Anja Šterbenc, Tanja Premru Sršen, Miha Lučovnik, Marijana Vidmar Šimic, Lili Steblovnik, Vesna Fabjan Vodušek, Mirjam Druškovič, Gorazd Kavšek, Mario Poljak and Andreja Trojner Bregar*

\title{
Usefulness of COVID-19 screen-and-test approach in pregnant women: an experience from a country with low COVID-19 burden
}

https://doi.org/10.1515/jpm-2020-0368

Received August 4, 2020; accepted October 10, 2020;

published online October 22, 2020

\section{Abstract}

Objectives: Information on the usefulness of screen-andtest strategies of pregnant women for severe acute respiratory syndrome coronavirus 2 (SARS-CoV-2) is lacking.

Methods: We retrospectively reviewed the Ljubljana Maternity Hospital database and searched for pregnant women, who were admitted to the hospital between March 15 and May 16, 2020, for a planned procedure or hospitalization. Their medical records were examined and SARS-CoV-2 test results were retrieved.

Results: During the two-month period analyzed, there were a total of 265 scheduled admissions of pregnant women to our hospital. Two hundred two (76.2\%) were tested for SARS-CoV-2 1 day prior to admission. All tested negative for SARS-CoV-2 RNA, regardless of having coronavirus disease 2019 (COVID-19)-compatible signs or symptoms $(\mathrm{n}=28)$ or not $(\mathrm{n}=174)$.

Conclusions: In a population with a low SARS-CoV-2 burden, usefulness of universal testing of pregnant women before admission to the hospital is limited. We recommend that obstetric units in regions with low SARS-CoV-2 burden

\footnotetext{
*Corresponding author: Andreja Trojner Bregar, MD, PhD, Department of Perinatology, Division of Obstetrics and Gynecology, University Medical Center Ljubljana, Šlajmerjeva 3, 1000 Ljubljana, Slovenia; and Faculty of Medicine, University of Ljubljana, Ljubljana, Slovenia, Phone: +38640299090, E-mail: andreja.trojner@kclj.si Anja Šterbenc and Mario Poljak, Institute of Microbiology and Immunology, Faculty of Medicine, University of Ljubljana, Ljubljana, Slovenia

Tanja Premru Sršen and Miha Lučovnik, Department of Perinatology, Division of Obstetrics and Gynecology, University Medical Center Ljubljana, Ljubljana, Slovenia; and Faculty of Medicine, University of Ljubljana, Ljubljana, Slovenia

Marijana Vidmar Šimic, Lili Steblovnik, Vesna Fabjan Vodušek, Mirjam Druškovič and Gorazd Kavšek, Department of Perinatology, Division of Obstetrics and Gynecology, University Medical Center Ljubljana, Ljubljana, Slovenia
}

enforce rational use of personal protective equipment and diligent screening protocols using targeted questionnaires, whereas SARS-CoV-2 laboratory testing should be performed only in screen-positives: those with high clinical suspicion of COVID-19 and/or suspected epidemiological history.

Keywords: COVID-19; pregnancy; SARS-CoV-2; screenand-test.

\section{Introduction}

In December 2019, a cluster of cases of pneumonia of unknown etiology was described in Wuhan, China. Shortly after, a novel coronavirus, named severe acute respiratory syndrome coronavirus 2 (SARS-CoV-2) was identified. Concerned by the alarming spread and severity of the SARS-CoV-2 infection, the World Health Organization (WHO) declared coronavirus disease 2019 (COVID-19) outbreak as a pandemic on March 11, 2020 [1].

Given the rapid spread of SARS-CoV-2 on a global scale, it was reasonable to speculate that some pregnant women could have also been infected. As a result, most obstetric units introduced some form of SARS-CoV-2 screening in pregnant women before and/or at the time of delivery. It is not known, however, whether such screening should also include universal laboratory testing for SARS-CoV-2. Data from New York City (USA) suggest that universal testing at the time of delivery could be beneficial, since up to $14 \%$ of asymptomatic pregnant women tested positive [2]. Usefulness of universal testing, however, always depends on pre-test probability and much lower positivity rate in pregnant women have been reported in populations with lower SARS-CoV-2 infection rates [3].

The first laboratory confirmed case of COVID-19 in Slovenia was reported on March 4, 2020 [4], followed by swift increase of new COVID-19 cases. On March 16, 2020, public transport was shut down and all educational institutions (preschools, schools, and universities) and public institutions such as museums, libraries, theatres, 
and sport facilities were closed. Non-essential medical procedures were cancelled, all non-essential shops and services were closed, and public gatherings were prohibited. In addition, international travel was restricted and national borders were completely closed. On March 29, 2020, the population mobility was further restricted to their home municipalities with strict police control. On April 1, 2020, our tertiary perinatal center implemented screening for SARS-CoV-2 in all women scheduled for a planned admission. Screening consisted of testing all women 1 day prior to scheduled admission and by using a questionnaire for identifying COVID-19-compatible signs and symptoms at the time of admission. Mobility restrictions within the country were lifted on May 1, 2020, followed by gradual reopening of health-care and dental services (May 9), reopening of public transport (May 11), and partial opening of preschools and schools (May 16). All of this ultimately led to Slovenia being the first country in Europe to officially declare the end of the first wave of the epidemic, effective as of May 31, 2020. As of July 30, 2020, the cumulative incidence of COVID-19 cases in Slovenia was 101.64 per 100,000 populations [5], which ranks Slovenia among European countries with the lowest reported COVID-19 burden. The aim of our study was to determine the usefulness of universal testing of asymptomatic women admitted to obstetric units in a population with a low SARS-CoV-2 burden.

\section{Methods}

In the period between April 1, 2020 (two weeks after SARS-CoV-2 epidemic was declared in Slovenia) and May 16, 2020 (1 day after the Slovenian Government officially proclaimed an end to the SARS-CoV-2 epidemic in Slovenia) all pregnant women scheduled for planned procedures, including planned Caesarean section, induction of labor, and scheduled hospitalization at the Department of Perinatology, University Medical Center Ljubljana, Slovenia, were screened for SARS-CoV-2 infection. Screening consisted of laboratory testing for SARS-CoV-2 1 day before admission as well as using a questionnaire survey for identifying COVID-19-compatible signs and symptoms at the time of admission.

One day before the scheduled admission, women were referred to one of the "COVID-19 entry points" (mostly at the Community Health Centre Ljubljana), where a nasopharyngeal swab was taken. The samples was tested for the presence of SARS-CoV-2 RNA using either of the two real-time reverse-transcription polymerase chain reaction (RT-PCR)-based approaches: fully automated cobas 6800 SARS-CoV-2 (Roche Molecular Systems, Branchburg, NJ, USA) [6] or LightMix Modular SARS and Wuhan CoV E-gene assays (TIB Molbiol, Berlin, Germany), following manufacturer's instructions [7]. Regardless of the SARS-CoV-2 test result, women were also screened at admission using detailed questionnaire. Women were specifically asked for any potential recent contacts with SARS-CoV-2-positive persons, history of traveling and the following signs/symptoms within the previous two weeks: elevated body temperature, coughing, sneezing, difficulty breathing, sore throat, diarrhea, vomiting, and loss of smell and taste. From 9 March 2020 onward, an isolation examination room was secured for all pregnant women who were screen-positive. A nasopharyngeal swab was collected by medical personnel wearing full personal protective equipment (PPE). Symptomatic women were provided with necessary medical care and were accommodated in the isolation room until SARS-CoV-2 RNA test results were received.

Women who were managed at an outpatient department and women who were admitted for spontaneous labor and delivery were screened for COVID-19-compatible signs and symptoms at the time of admission and only symptomatic women were subsequently tested.

Hospital policies recommended universal use of a surgical mask for clinicians, other healthcare personnel and patients. For patients without COVID-19-related symptoms, clinicians followed general protective precautions (e.g., wearing a surgical mask). A visor with protective cap (or alternative mask IIR, protective cap and protective glasses), protective coat, extended (sterile) nitrile gloves were used, if necessary. Medical staff wore PPE during testing of symptomatic patients and their management until SARS-CoV-2-negative test results were received and continued to do so if pregnant women tested positive for SARS-CoV-2 RNA. While working with SARS-CoV-2-positive pregnant women clinicians, midwifes and other healthcare personnel used FFP3 masks, protective caps, tight-fitting protective glasses (or alternative face masks with filter and cap or VersAflo), water-repellent coats with cuffs, and extended (sterile) nitrile gloves.

In June 2020, we reviewed the Ljubljana Maternity Hospital medical database and searched for pregnant women, who were admitted to the hospital between March 15 and May 16, 2020, for a scheduled procedure or hospitalization. Their medical records were examined, and SARS-CoV-2 RNA results were retrieved. In addition, we reviewed medical records and SARS-CoV-2 RNA test results of hospitalized obstetric patients who developed symptoms consistent with a respiratory infection during hospitalization.

This quality improvement project does not meet the definition of human subject research. Hence, according to Slovenian legislation, a review by the institutional review/ethical board was not required in order to analyze the data.

\section{Results}

During the period analyzed, there was a total of 4,043 hospitalizations and/or outpatient visits at our center. There were 265 scheduled admissions and nasopharyngeal swabs for SARS-CoV-2 RNA testing were obtained in 202 (76.2\%) women on the day before their scheduled admission. At admission, 232 (87.5\%) women scheduled for a procedure/hospitalization presented with no COVID19-compatible signs or symptoms. Of these, 174 (75.0\%) were tested for SARS-CoV-2 RNA the day before and none tested positive.

Of the 33 pregnant women with a planned procedure or hospitalization, nasopharyngeal swabs were obtained from 28 women who presented with symptoms suggesting a respiratory infection (e.g., fever, sore throat, loss of taste 


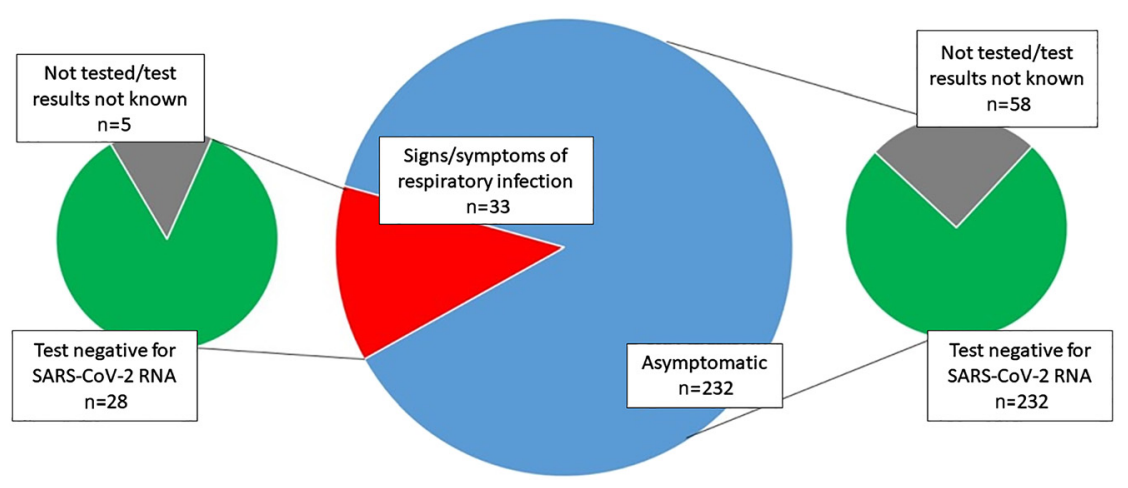

Figure 1: The study population.

and smell, fatigue, cough). All of them were negative for SARS-CoV-2 RNA (Figure 1).

During the study period, seven hospitalized women developed fever or respiratory symptoms post-partum. None of them tested positive for SARS-CoV-2 RNA (Figure 1).

Eight SARS-CoV-2-RNA-positive obstetrical patients were managed at our institution during the two-month period analyzed. All of them were admitted with previously laboratory confirmed active COVID-19. All eight women were regularly monitored at a COVID-19 outpatient unit. Three women are still pregnant (all became negative for SARS-CoV-2 RNA in follow-up), whereas five women delivered vaginally and all newborns were term, normal weight healthy babies. Only two deliveries needed to be carried out in a separated COVID-19 labor ward due to a positive SARS-CoV-2 RNA status at the time of delivery. On the other hand, three patients that tested positive for SARS-CoV-2 RNA during pregnancy became SARS-CoV2-RNA-negative (e.g., had two consecutive SARS-CoV-2 RNA negative nasopharyngeal swab samples) before admission to the labor ward, hence no additional precautionary procedures were implemented. No health care workers that were involved in management of SARS-CoV2-positive pregnant women have been infected with SARS-CoV-2 during the observation period.

\section{Discussion}

Between March 15 and May 16, 2020, a total of 1,252 individuals were newly diagnosed with COVID-19 in Slovenia [8]. Nevertheless, progressive and drastic lockdown measures implemented by the Slovenian government significantly impacted the burden of the disease in April and May 2020. Moreover, a study on a probability-based sample of the Slovenian population performed in late April 2020 showed a very low prevalence of active COVID-19 infections (2/1,366; prevalence $0.15 \%, 95 \%$ Bayesian confidence interval [CI] 0.03-0.47\%) [9]. With a total of 122 deaths as of August 4, the mortality rate of COVID-19 in our country is estimated at 59.01 per million [10].

The main finding of our study is that universal testing for SARS-CoV-2 in women admitted for delivery is very unlikely to be useful in areas/countries with low COVID-19 burden. During the two-month intensive surveillance, we have not identified a single new SARS-CoV-2-positive case among asymptomatic and even symptomatic pregnant women scheduled for a planned procedure at our institution. In addition, none of the seven patients that developed symptoms consistent with a respiratory infection during hospitalization tested positive for SARS-CoV-2 RNA, suggesting infection with other respiratory pathogens. Our results are in contrast to those by Sutton et al., who reported that approximately $13.5 \%$ of asymptomatic women presenting for childbirth in New York City test positive for SARS-CoV-2 [2]. Conversely, our findings are more in line with the data from Southern Connecticut, where a low prevalence $(<3 \%)$ of SARS-CoV-2 among asymptomatic pregnant population was found [11]. Similarly, because none of the asymptomatic pregnant women tested at the Cedars-Sinai Medical Center labor and delivery unit (Los Angeles, USA) were shown to be SARS-CoV-2-positive, universal SARS-CoV-2 RNA testing of pregnant women was discontinued; however, this may change if local infection rates increase [3]. Data from Madrid (Spain), one of the most severely affected European cities, also suggest that the effectiveness of universal SARS-CoV-2 screening at admission to labor depends on the current epidemiological situation in the region [12]. Herraiz et al. found that during the post-peak period, only $0.5 \%$ of asymptomatic pregnant women tested positive for SARS-CoV-2 RNA at admission to labor [12]. It is possible that the observed discrepancies are a result of markedly diverse SARS-CoV-2 incidence rates 
across various geographical regions, as well as variations in basic reproduction number $\left(\mathrm{R}_{0}\right)$ of SARS-CoV-2 among different populations due to implementation or lack of mitigation strategies to contain the epidemic [13].

With limited information on the impact of COVID-19 on pregnancy as well as perinatal and neonatal outcomes during the early stages of COVID-19 pandemic [14], initial shortages of PPE and lack of international consensus guidelines on management of obstetric patients, each medical center had to adjust their protocols according to the national guidance (if applicable). On April 28, 2020, FIGO (The International Federation of Gynecology and Obstetrics) and allied partners published global interim guidance on COVID-19 during pregnancy and puerperium that included management guidance for four main settings of pregnancy, including a) ambulatory antenatal care in the outpatient clinics; b) management in the setting of the obstetrical triage; c) intrapartum management; and d) postpartum management and neonatal care [15]. These guidelines will be especially useful in case of a worsening epidemiological situation and potential second epidemic wave. A universal testing approach for all pregnant women might indeed identify additional COVID-19 cases, help determine hospital isolation practices and guide neonatal care as well as the use of PPE during obstetric management [2]; however, its practical usefulness in various epidemiological settings remains to be seen. Thus careful balance is needed in order to prevent psychological burden and overtesting of this vulnerable population, especially in countries with a low COVID-19 burden.

Our study has several limitations. We analyzed a relatively short time period (two months) and included data from a single center only. However, our institution is the biggest tertiary perinatal center in Slovenia, with almost $1 / 3$ of all deliveries in the country (around 5,500 deliveries per year), accepting also in utero transfers $<34$ gestational weeks as well as otherwise high-risk pregnant women from all other obstetrical units within our country. Despite most probably being representative, our data still cannot be directly generalized to the whole country. Regardless of having a clear protocol for management of women scheduled for a planned procedure or hospitalization, $25.0 \%$ (58/232) of eligible women were not tested for SARS-CoV-2, suggesting breach of protocol occurred in a significant proportion of cases. These women were mostly admitted to our institution before their planned date of admission or were tested for SARS-CoV-2 at another institution. Identifying such inaccuracies is of utmost importance for successful handling of future COVID-19 outbreaks in our country.

\section{Conclusions}

Results from our two-month SARS-CoV-2 testing of asymptomatic pregnant women before scheduled admission suggest little benefit of such approach in areas with low COVID-19 burden. Instead, diligent questionnairebased screening for COVID-19-compatible clinical signs and symptoms with laboratory testing restricted to screenpositive women seems a more rational approach in low prevalence populations. Nevertheless, obstetricians should closely monitor the national/regional status of COVID-19 epidemic, as the epidemiological situation may change abruptly and drastically.

Research funding: None declared.

Author contributions: All have contributed significantly to the work and have seen and approved the final version of the manuscript.

Competing interests: The authors declare no conflict of interest.

Ethical approval: This quality improvement project does not meet the definition of human subject research. Hence, according to Slovenian legislation, a review by the Institutional Review/Ethical Board was not required in order to analyze the data.

\section{References}

1. Spiteri G, Fielding J, Diercke M, Campese C, Enouf V, Gaymard A, et al. First cases of coronavirus disease 2019 (COVID-19) in the WHO European region, 24 January to 21 February 2020. Euro Surveill 2020;25:2000178.

2. Sutton D, Fuchs K, D’Alton M, Goffman D. Universal screening for SARS-CoV-2 in women admitted for delivery. N Engl J Med 2020; 382:2163-4.

3. Naqvi M, Burwick RM, Ozimek JA, Greene NH, Kilpatrick SJ, Wong MS. Severe acute respiratory syndrome coronavirus 2 (SARS-CoV-2) universal testing experience on a Los Angeles labor and delivery unit. Obstet Gynecol 2020. https://doi.org/10.1097/ AOG.0000000000003987 [Epub ahead of print].

4. National Institute of Public Health. Coronavirus disease COVID-19 [Internet]. Available from: https://nijz.si/en/coronavirus-diseasecovid-19 [Accessed 29 Jun 2020].

5. Statista. Incidence of coronavirus (COVID-19) cases in Europe as of July 30, 2020, by country [Internet]. Available from: https://www. statista.com/statistics/1110187/coronavirus-incidence-europeby-country/ [Accessed 3 Aug 2020].

6. Poljak M, Korva M, Knap Gašper N, Fujs Komloš K, Sagadin M, Uršič T, et al. Clinical evaluation of the cobas SARS-CoV-2 test and a diagnostic platform switch during 48 hours in the midst of the COVID-19 pandemic. J Clin Microbiol 2020;58: e00599-20. 
7. Corman VM, Landt O, Kaiser M, Molenkamp R, Meijer A, Chu DK, et al. Detection of 2019 novel coronavirus (2019-nCoV) by realtime RT-PCR. Euro Surveill 2020;25. 2000045. https://doi.org/10. 2807/1560-7917.ES.2020.25.3.2000045.

8. Republika Slovenija. Koronavirus (SARS-CoV-2) [Internet]. Available from: https://www.gov.si/teme/koronavirus-sars-cov2/ [Accessed 29 Jun 2020].

9. Maver Vodičar P, Oštrbenk Valenčak A, Zupan B, Avšič Županc T, Kurdija S, Korva M, et al. Low prevalence of active COVID-19 in Slovenia: a nationwide population study on a probability-based sample. Clin Microbiol Infect 2020. https://doi.org/10.1016/j. cmi.2020.07.013 [Epub ahead of print].

10. Statista. Coronavirus (COVID-19) deaths worldwide per one million population as of August 4, 2020, by country [Internet]. Available from: https://www.statista.com/statistics/1104709/ coronavirus-deaths-worldwide-per-million-inhabitants/ [Accessed 4 Aug 2020].

11. Campbell KH, Tornatore JM, Lawrence KE, Illuzzi JL, Sussman LS, Lipkind HS, et al. Prevalence of SARS-CoV-2 among patients admitted for childbirth in southern Connecticut. J Am Med Assoc 2020;323:2520-2.

12. Herraiz I, Folgueira D, Villalaín C, Forcén L, Delgado R, Galindo A. Universal screening for SARS-CoV-2 before labor admission during Covid-19 pandemic in Madrid. J Perinat Med 2020;48: 981-4.

13. Anderson RM, Heesterbeek $\mathrm{H}$, Klinkenberg D, Hollingsworth TD. How will country-based mitigation measures influence the course of the COVID-19 epidemic? Lancet 2020;395: 931-4.

14. Trippella G, Ciarcià M, Ferrari M, Buzzatti C, Maccora I, Azzari C, et al. COVID-19 in pregnant women and neonates: a systematic review of the literature with quality assessment of the studies. Pathogens 2020;9:E485.

15. Poon LC, Yang H, Kapur A, Melamed N, Dao B, Divakar H, et al. Global interim guidance on coronavirus disease 2019 (COVID-19) during pregnancy and puerperium from FIGO and allied partners: information for healthcare professionals. Int J Gynaecol Obstet 2020;149:273-86. 